\title{
Genetic Variability among Released Sorghum [Sorghum bicolor (L.) Moench] Varieties in West Hararghe Zone
}

\author{
Gebeyehu Chala, Bulti Tesso (PhD) and Dagnachew Lule (PhD) \\ Oromia Agricultural Research Institute, Mechara Agricultural Research Center, P.O.Box, 19
}

\begin{abstract}
The Experiment was conducted at Mechara Agricultural Research Center on station and Miesso sub-site during 2017 cropping season. The objective was to estimate the Phenotypic and genotypic variability among released sorghum varieties. Data was collected and analyzed based on eight traits like: Days to flowering(days), Days to maturity(days), Grain filling period(days), Grain filling rate(\%), Head weight(gram), Hundred seed weight(gram), Stand count at harvest(number) and Grain yield(ton/ha). These traits and variances were recorded on 22 genotypes of sorghum. Results of statistical analysis showed that all traits had higher phenotypic variance and phenotypic coefficient of variation than genotypic variance and genotypic coefficient of variation, which indicated the influence of environment was least, and can be exploited in breeding programs. The genotypes exhibited varying degrees ratios of heritability for most traits. Such traits were responded positively to selection due to high broad sense heritability estimates. These data demonstrated high diversity for the traits studied of genotypes used and finally Baji and Birmash was recommended for further demonstration on farmer's field.
\end{abstract}

Keywords: GCV, PCV, Sorghum, Variability, Yield.

DOI: $10.7176 / \mathrm{JNSR} / 9-1-05$

\section{Introduction}

Sorghum (Sorghum bicolor L. Moench) is the second most important dry land crop for semi-arid tropics. It is grown in an area of 38 million hectares and with annual grain production of about 58 million tones and the productivity average reaches $1.5 \mathrm{ton} / \mathrm{ha}$. (FAOSTAT, 2014). Although sorghum has an ability to tolerate many of environment stress, but it is affected by drought stress at the post flowering stage of growth (Hall, 1993). Genetic variability for agronomic traits is a key component of breeding programs for broadening the gene pool of crops (Ramirez and Kelly, 1998). Heritability is measure of the phenotypic variance attributable to genetic causes and has predictive function in plant breeding. It provides information on the extent to which a particular genetic- morphological traits can be transmitted to successive generations, and influences the choice of selection procedures used by the plant breeder to decide which selection methods would be most useful to improve the trait, to predict gain from selection and to determine the relative importance of genetic effects (Kebede et al., 2001). Falconer and Mackay, (1997) reported that the most important function of heritability in genetic studies of quantitative traits is its predictive role to indicate the reliability of phenotypic value as guide to breeding value. $\mathrm{Xu}$ et al. (2000) illustrated that traits with high heritability can easily be fixed with simple selection resulting in quick progress, but it has been emphasized that heritability alone has no practical importance without genetic advance. High genetic advance with high heritability estimates offers the most suitable condition for selection. High heritability of population is not enough to insure a final high yield/ plant without a high yield/plant originally in the base population or first cycle of selection of a scheme (Rosenow and Clark, 1995). Tadesse et al. (2011) indicated that the most traits had relatively higher genotypic and phenotypic variance components and little difference between PCV and indicating their variation has a genetic origin can be exploited for further breeding programs. Tesfamicheal et al. (2011) found high magnitude of PCV and GCV for all agronomic traits studied. Individual and the combined analysis indicated that most traits had higher genotypic and phenotypic variance components than the environmental variance estimates, which is indicative that traits expression in this sorghum population was genetic and can be exploited in breeding programs (Xu et al., 2000). Evaluation of components of variation and heritability for many traits will be facilitate improvement of crops, such as sorghum thus the objectives were to evaluate the variation in genetic morphology and genetic- physiology traits in order to improve the yield of sorghum plants.

\section{Materials and Methods}

\subsection{Description of Experiment Site}

Sorghum genotypes were planted during 2017 cropping season at Mechara Agricultural Research Center and Miesso sub-site in order to analyze some genetic parameters for yield and yield related components of sorghum varieties. 
Table 1.Agro-ecological features of the experimental locations.

\begin{tabular}{|c|c|c|c|c|c|c|c|}
\hline \multirow[b]{2}{*}{ Locations } & \multirow{2}{*}{$\begin{array}{l}\text { Altitude } \\
\text { (m.a.s.l) }\end{array}$} & \multirow{2}{*}{$\begin{array}{l}\text { Ave. } \\
\text { RF(mm) }\end{array}$} & \multirow[b]{2}{*}{ Soil Type } & \multicolumn{2}{|c|}{ Geographic coordinates } & \multicolumn{2}{|c|}{$\begin{array}{l}\text { Ave. } \\
\text { Temp. }\left({ }^{\circ} \mathrm{C}\right)\end{array}$} \\
\hline & & & & Latitude & Longitude & Max. & Min. \\
\hline Mechara & 1760 & 871 & Sandy loam & $8^{\circ} 36^{\prime} \mathrm{N}$ & $40^{\circ} 18^{\prime} \mathrm{E}$ & 23.4 & 8.9 \\
\hline Mieso & 1470 & 856.8 & Vertisoil & $16^{\circ} 06^{\prime} \mathrm{N}$ & $37^{\circ} 8^{\prime} \mathrm{E}$ & 35.0 & 8.3 \\
\hline
\end{tabular}

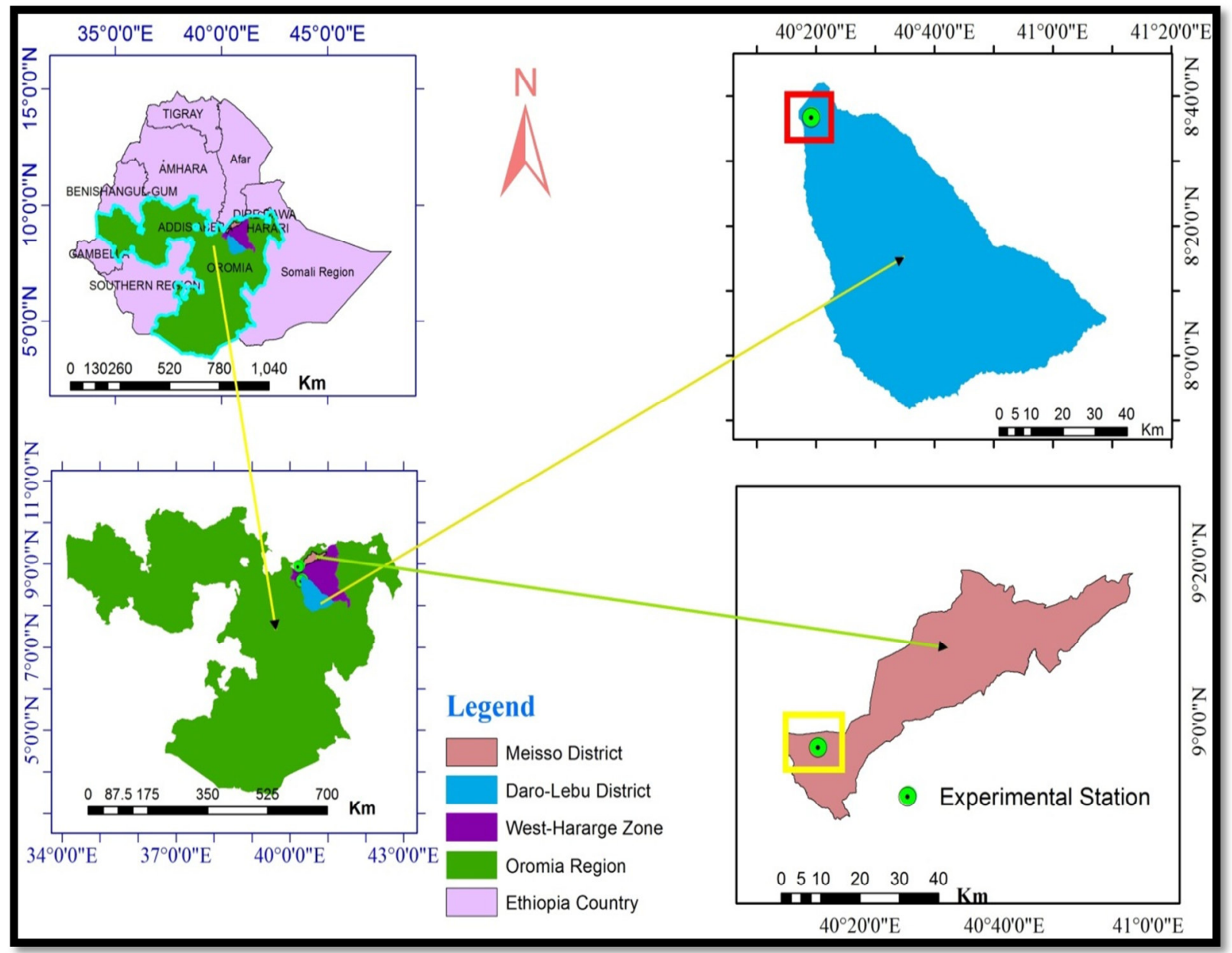

Figure 1. Map of the study Area

\subsection{Plant Materials}

The experimental plant materials comprised of 21 sorghum varieties excluding local check released from different research centers of Ethiopian sorghum production. One local check was included at each location. The detailed information about the materials is presented in Table 2. 
Table 2. Description of different sorghum varieties tested at five locations.

\begin{tabular}{llllll}
\hline $\begin{array}{l}\# \\
\text { No }\end{array}$ & Varieties & Pedigree & $\begin{array}{l}\text { Year } \\
\text { Release }\end{array}$ & $\begin{array}{l}\text { Adaptation } \\
\text { area } \\
\text { (m.a.s.l.) }\end{array}$ & $\begin{array}{l}\text { Seed Breeder/ } \\
\text { Maintainer }\end{array}$ \\
\hline 1 & Baji & 85 MW 5334 & 1996 & $1600-1900$ & MARC/EIAR \\
2 & Birmash & NA & 1989 & $1600-1900$ & MARC/EIAR \\
3 & Geremew & 87 BK -4122 & 2007 & $1600-1900$ & MARC/EIAR \\
4 & Lalo & BRC-245 & 2006 & $>1600$ & BARC/OARI \\
5 & Teshale & 3443-2-0P & 2002 & $1450-1850$ & SRARC/ARARI \\
& & & & & and MARC/EIAR \\
6 & Melkam & WSV 387 & 2009 & $<1600$ & MARC/EIAR \\
7 & Gobiye & P-9401 & 1999 & $<1850$ & MARC/EIAR \\
8 & Abshir & P-9403 & 2000 & $<1850$ & MARC/EIAR \\
9 & Dagim & IS10892XRS/R-20-8614-2 X IS & 2011 & $1600-1900$ & SRARC \\
10 & IS9302 & NA & 1981 & $1600-1900$ & MARC/EIAR \\
11 & ESH-1 & P-9501 A X ICSR14 & 2009 & $<1600$ & MARC/EIAR \\
12 & Birhan & Key\#8566 & 2002 & $<1850$ & SRARC/ARARI \\
13 & Gambella- & NA & 1981 & $1450-1850$ & MARC/EIAR \\
& 1107 & & & & \\
14 & Emahoy & Pw01-092 & 2007 & $1600-1900$ & PARC/EIAR \\
15 & Dekeba & ICSR 24004 & 2012 & $<1600$ & MARC/EIAR \\
16 & Chemeda & Acc-BCC-5 & 2013 & $>1600$ & BARC/OARI \\
17 & Local & - & - & - & Farmers \\
18 & 07MW6035 & (89MW4122*85MW5552)*85MW5340 & 2016 & $1600-1900$ & MARC/EIAR \\
19 & 07MW6002 & (89MW4122*85MW5552)*85MW5340 & 2016 & $1600-1900$ & MARC/EIAR \\
20 & Assosa_1 & Bambasi \# & 2015 & $1500-1850$ & AARC \\
21 & Adukara & NA & 2015 & $1500-1850$ & AARC \\
22 & 07MW6052 & (89MW4122*85MW5552)*85MW5340 & 2016 & $1600-1900$ & MARC/EIAR \\
\hline EIARARA & & & \\
\end{tabular}

EIAR=Ethiopian Institute of Agricultural Research, MARC $=$ Melkasa Agricultural Research Center, BARC $=$ Bako Agricultural Research Center, JARC $=$ Jimma Agricultural Research Center, SRARC $=$ Sirinka Agricultural Research Center, ARARI=Amhara Regional Agricultural Research Institute, PARC $=$ Pawe Agricultural Research Center AARC $=$ Assosa Agricultural Research Center, NA= Not Available.

\subsection{Experimental Design and Plot Arrangements}

The trial was laid out in randomized complete block design (RCBD) with three replications. The experimental plots consist of two (2) rows, each $5 \mathrm{~m}$ in length with $75 \mathrm{~cm}$ row to row and $15 \mathrm{~cm}$ plant-to-plant spacing. The total area of each plot size was two rows of $75 \mathrm{~cm}$ between rows and five meter row length $\left(7.5 \mathrm{~m}^{2}\right)$. Seeds were sown by hand drilling. A seed rate of $12 \mathrm{~kg} / \mathrm{ha}$ and $13.5 \mathrm{gm} / \mathrm{plot}$ was used as per the recommendation for row planting. Thinning was done two weeks after emergence to adjust plant to plant spacing. NPS fertilizer was applied according to recommended rate. During planting, $100 \mathrm{~kg} / \mathrm{ha}$ of NPS was applied in the seed furrow at planting. Urea was applied as top dressing in split application at the rate of $50 \mathrm{~kg} / \mathrm{ha}$ at knee height stage. The field was kept free of weeds during the period of the experiment. All other recommended agronomic management practices such as land preparation and insect pest control were done.

\subsection{Data Collection and Analysis}

Data like Days to flowering, Days to maturity, Grain filling period, Grain filling rate, Head weight, hundred seed weight, Stand count at harvest and Grain yield data were collected and analyzed by Genstat $16^{\text {th }}$ edition and LSD mean separation was used at 5\% probability level to test the significance of the treatments.

\subsubsection{Statistical analysis}

Based on the ANOVA, genotypic coefficient of variation (GCV), phenotypic coefficient of variation (PCV), heritability in the broad sense and genetic advance (GA) were estimated as follows: GCV and PCV (\%) were computed according to the formula suggested by Burton and Devane (1953) as follows:

Genotypic variance $\left(\delta^{2} \mathrm{~g}\right)=\frac{(M S g-M S e)}{r}$

Phenotypic variance $\left(\delta^{2} \mathrm{p}\right)=\delta^{2} \mathrm{~g}+\delta^{2} \mathrm{e}$

$\mathrm{GCV}=\frac{\sqrt{\sigma^{2} \mathrm{~g}}}{\mathrm{y}} \times 100 \%$,

$\mathrm{PCV}=\frac{\sqrt{\sigma^{2} \mathrm{ph}}}{\mathrm{y}} \times 100 \% \quad$ Where, 
$\delta^{2} \mathrm{p}=$ phenotypic variation

$\delta^{2} \mathrm{~g}=$ genotypic variation

$\mathrm{y}=$ Grand mean of the character studied.

Heritability in the broad sense $\left(\mathrm{h}^{2} \mathrm{~B}\right)$ was estimated in each season separately from the ANOVA according to Johnson et al. (1955) by the formula:

$\mathrm{h}^{2} \mathrm{~B}=\frac{\sigma^{2} \mathrm{~g}}{\sigma^{2} p h} \times 100 \%$

Expected GA was estimated by the formula of Allard (1960) as follows:

$\mathrm{GA}=\frac{k \sigma^{2} \mathrm{~g}}{\sigma^{2} p h y} \times 100 \%$

Where, $\sigma^{2} \mathrm{~g}$ is the genotypic variance, $\sigma^{2} \mathrm{ph}$ is the phenotypic variance, $\mathrm{y}$ is the general mean of a character and $\mathrm{k}$ is the selection differential which has the value of 2.06 as defined by Lush (1949) at selection intensity of $5 \%$. The phenotypic correlation between grain yields, days to $50 \%$ flowering, plant height, number of heads/plot, 100 -seed weight traits were estimated.

\section{Results and Discussion}

The analysis of Variance (ANOVA) showed that the mean squares due to genotypes were highly significant (P $<0.01$ ) for all of the traits recorded. (Table 3), indicating the existence of adequate variations among the tested genotypes. The value of coefficient of variation for most of the traits indicated that good precision of the experiment. Haile et al. (2016) studied 974 sorghum landraces and reported significant differences in days to 50\% flowering, days to $90 \%$ maturity, plant height and thousand kernel weights. Mihret et al. (2015), similar to the present study, observed highly significant variation $(\mathrm{P}<0.01)$ for the agronomic traits (days to flowering, days to maturity, plant height, hundred seed weights and grain yield) (Table 3 ).

Table 3. Mean sum of square for 22 quantitative characters in sorghum varieties.

\begin{tabular}{llllllllll}
\hline Source of Var. & DF & DM & PH & GFP & GFR & SCH & HW & HSW & GY \\
\hline Replication (2) & 84.0 & 663 & 82 & 278.6 & 1451 & 2.4 & 442.3 & 48.24 & 0.42 \\
Treatments (21) & $371.7^{* *}$ & $501^{* *}$ & $5034^{*}$ & $135.5^{*}$ & $293^{*}$ & $108.9^{* *}$ & $562.4^{* *}$ & $25.51^{* *}$ & $5.13^{*}$ \\
Error (306) & 185.2 & 1255 & 1049 & 682.3 & 1653 & 236.4 & 625.1 & 38.38 & 1.424 \\
LSD & 9.78 & 25.45 & 23.27 & 18.77 & 29.22 & 11.05 & 17.97 & 4.45 & 0.86 \\
SE + C & 13.61 & 35.42 & 32.39 & 26.12 & 40.66 & 15.38 & 25.00 & 6.19 & 1.19 \\
CV & 14.7 & 24.0 & 17.7 & 39.0 & 26.0 & 35.2 & 37.2 & 19.5 & 26.0 \\
\hline
\end{tabular}

NB:DF=Days to flowering(days), DM=Days to maturity(days), $\mathrm{PH}=\mathrm{Plant}$ height(cm), GFP=Grain filling period(days), GFR $=$ Grain filling rate(days), $\mathrm{SCH}=$ Stand count at harvest(number), $\mathrm{HW}=$ Head weight(gram), $\mathrm{HSW}=$ Hundred seed weight(gram), GY= Grain yield(ton/ha), Numbers in parenthesis indicates degrees of freedom, $\mathrm{SE}=$ Standard error, $\mathrm{CV}=$ Coefficient of variation.

\subsection{Comparison of Phenotypic Performance of the Genotypes}

3.1.1. Crop phenology

The genotypes showed considerable variation in plant phenology. The mean in days to flowering at individual location was ranged from 67 to 118 days at Mechara and 79 to 112 days at Miesso. The mean plant height was ranged from 118 to 344 days at Mechara and 117.7 to 273.7 days at Miesso, The mean grain filling period was ranged from 43 to 62.67 days at Mechara and 38 to 99 days at Miesso, The mean grain filling rate was ranged from 23.06 to 98.11 days at Mechara and 10.47 to 52.39 days at Miesso and Grain yield ranged from 0.93 to 5.12 ton/ha at Mechara and 0.1 to 2.5 ton/ha at Miesso (Table 4 and 5). Partially agreed results for days to flowering was also reported by Amsalu and Endashaw (2012) for Tigray and Wollo genotypes and Haile et al.(2016) for Northern Ethiopian sorghum landraces. 
Table 4. Mean square values of yield and yield related traits of sorghum varieties at Mechara

\begin{tabular}{|c|c|c|c|c|c|c|c|c|c|}
\hline Varieties & $\mathrm{DF}$ & $\mathrm{DM}$ & $\mathrm{PH}$ & $\mathrm{SCH}$ & GFP & GFR & HW & HSW & GY \\
\hline Baji & $74.67^{\mathrm{d}-\mathrm{g}}$ & $137.0^{\text {de }}$ & $173.7^{\mathrm{ef}}$ & $38.00^{\mathrm{a}-\mathrm{d}}$ & $58.33^{\mathrm{a}-\mathrm{c}}$ & $66.05^{b-f}$ & $84.33^{b-f}$ & $33.14^{b}$ & $3.863^{\mathrm{a}-\mathrm{c}}$ \\
\hline Birmash & $94.00^{\mathrm{a}-\mathrm{f}}$ & $138.0^{\mathrm{de}}$ & $201.0^{\mathrm{d}}$ & $50.00^{\mathrm{ab}}$ & $58.67^{\mathrm{a}-\mathrm{c}}$ & $70.32^{b-f}$ & $87.67^{\mathrm{b}-\mathrm{f}}$ & $35.55^{\mathrm{b}}$ & $4.159^{\mathrm{ab}}$ \\
\hline Geremew & $87.33^{b-g}$ & $139.3^{\mathrm{de}}$ & $162.0^{\mathrm{fg}}$ & $37.33^{\mathrm{a}-\mathrm{d}}$ & $46.00^{\mathrm{d}-\mathrm{f}}$ & $48.83^{\mathrm{e}-\mathrm{h}}$ & $81.67^{\mathrm{d}-\mathrm{f}}$ & $29.38^{\mathrm{b}}$ & $2.271^{\mathrm{e}-\mathrm{h}}$ \\
\hline Lalo & $111.67^{\mathrm{ab}}$ & $145.3^{\mathrm{cd}}$ & $344.0^{\mathrm{a}}$ & $37.67^{\mathrm{a}-\mathrm{d}}$ & $33.67^{\mathrm{g}}$ & $60.72^{c-f}$ & $99.33^{\mathrm{a}-\mathrm{e}}$ & $31.96^{\mathrm{b}}$ & $2.110^{\mathrm{f}-\mathrm{h}}$ \\
\hline Teshale & $77.00^{\mathrm{c}-\mathrm{g}}$ & $118.7^{\mathrm{f}}$ & $193.0^{\mathrm{d}}$ & $44.00^{\mathrm{a}-\mathrm{c}}$ & $51.00^{\mathrm{b}-\mathrm{e}}$ & $84.91^{\mathrm{a}-\mathrm{c}}$ & $58.33^{\text {f-h }}$ & $31.28^{\mathrm{b}}$ & $4.331^{\mathrm{ab}}$ \\
\hline Melkam & $73.33^{\mathrm{e}-\mathrm{g}}$ & $120.0^{\mathrm{f}}$ & $149.3^{\text {gh }}$ & $43.33^{\mathrm{a}-\mathrm{c}}$ & $48.67^{\mathrm{c}-\mathrm{e}}$ & $75.84^{\mathrm{a}-\mathrm{e}}$ & $62.67^{\mathrm{e}-\mathrm{g}}$ & $36.12^{\mathrm{b}}$ & $3.717^{\mathrm{a}-\mathrm{d}}$ \\
\hline Gobiye & $66.67^{\text {fg }}$ & $119.0^{\mathrm{f}}$ & $118.3^{\mathrm{j}}$ & $34.33^{\mathrm{b}-\mathrm{e}}$ & $53.67^{\mathrm{a}-\mathrm{d}}$ & $42.48^{\mathrm{f}-\mathrm{h}}$ & $42.33^{\mathrm{gh}}$ & $32.91^{\mathrm{b}}$ & $2.280^{\mathrm{e}-\mathrm{h}}$ \\
\hline Abshir & $86.33^{b-g}$ & $119.7^{\mathrm{f}}$ & $145.7^{\mathrm{h}}$ & $36.00^{\mathrm{b}-\mathrm{d}}$ & $52.33^{\mathrm{a}-\mathrm{e}}$ & $44.68^{\mathrm{f}-\mathrm{h}}$ & $48.33^{\mathrm{f}-\mathrm{h}}$ & $33.28^{\mathrm{b}}$ & $2.325^{\mathrm{d}-\mathrm{h}}$ \\
\hline Dagim & $81.33^{\mathrm{c}-\mathrm{g}}$ & $138.0^{\mathrm{de}}$ & $193.3^{d}$ & $38.33^{\mathrm{a}-\mathrm{d}}$ & $55.00^{\mathrm{a}-\mathrm{d}}$ & $47.21^{\mathrm{f}-\mathrm{h}}$ & $82.33^{\mathrm{d}-\mathrm{f}}$ & $32.30^{\mathrm{b}}$ & $2.615^{\mathrm{c}-\mathrm{g}}$ \\
\hline IS9302 & $80.67^{\mathrm{c}-\mathrm{g}}$ & $139.7^{\mathrm{de}}$ & $199.7^{\mathrm{d}}$ & $42.67^{\mathrm{a}-\mathrm{c}}$ & $59.00^{\mathrm{a}-\mathrm{c}}$ & $58.80^{\mathrm{c}-\mathrm{f}}$ & $83.33^{c-f}$ & $34.07^{\mathrm{b}}$ & $3.490^{b-f}$ \\
\hline ESH-1 & $69.67^{\mathrm{fg}}$ & $120.3^{\mathrm{f}}$ & $179.0^{\mathrm{e}}$ & $31.00^{\mathrm{c}-\mathrm{e}}$ & $50.67^{\mathrm{b}-\mathrm{e}}$ & $46.02^{\mathrm{f}-\mathrm{h}}$ & $78.00^{\mathrm{d}-\mathrm{g}}$ & $32.74^{\mathrm{b}}$ & $2.333^{\mathrm{d}-\mathrm{h}}$ \\
\hline Birhan & $64.67^{\mathrm{g}}$ & $119.7^{\mathrm{f}}$ & $140.0^{\mathrm{hi}}$ & $43.67^{\mathrm{a}-\mathrm{c}}$ & $55.00^{\mathrm{a}-\mathrm{d}}$ & $44.19^{\mathrm{f}-\mathrm{h}}$ & $24.67^{\mathrm{h}}$ & $31.50^{\mathrm{b}}$ & $2.429^{\mathrm{d}-\mathrm{g}}$ \\
\hline Gambela-1107 & $75.33^{\mathrm{d}-\mathrm{g}}$ & $119.7^{\mathrm{f}}$ & $178.0^{\mathrm{e}}$ & $38.00^{\mathrm{a}-\mathrm{d}}$ & $42.00^{\mathrm{e}-\mathrm{g}}$ & $98.11^{\mathrm{a}}$ & $63.67^{\mathrm{d}-\mathrm{g}}$ & $32.59^{\mathrm{b}}$ & $4.121^{\mathrm{ab}}$ \\
\hline Emahoy & $71.33^{\mathrm{e}-\mathrm{g}}$ & $126.3^{\mathrm{ef}}$ & $222.7^{\mathrm{c}}$ & $54.67^{\mathrm{a}}$ & $55.00^{\mathrm{a}-\mathrm{d}}$ & $89.18^{\mathrm{ab}}$ & $50.00^{\mathrm{f}-\mathrm{h}}$ & $44.91^{\mathrm{a}}$ & $4.871^{\mathrm{ab}}$ \\
\hline Dekeba & $81.33^{\mathrm{c}-\mathrm{g}}$ & $120.7^{\mathrm{f}}$ & $127.7^{\mathrm{ij}}$ & $35.67^{\mathrm{b}-\mathrm{d}}$ & $45.67^{\mathrm{d}-\mathrm{f}}$ & $89.35^{\mathrm{ab}}$ & $65.67^{\mathrm{d}-\mathrm{g}}$ & $33.68^{\mathrm{b}}$ & $4.090^{\mathrm{ab}}$ \\
\hline Chemeda & $103.67^{\mathrm{a}-\mathrm{c}}$ & $167.0^{\mathrm{ab}}$ & $330.3^{b}$ & $32.67^{\mathrm{b}-\mathrm{e}}$ & $57.33^{\mathrm{a}-\mathrm{c}}$ & $63.13^{b-f}$ & $122.33^{\mathrm{ab}}$ & $31.58^{\mathrm{b}}$ & $3.593^{\mathrm{b}-\mathrm{e}}$ \\
\hline Local & $90.33^{b-g}$ & $139.0^{\mathrm{de}}$ & $140.0^{\mathrm{hi}}$ & $20.33^{\mathrm{c}-\mathrm{e}}$ & $36.33^{\mathrm{fg}}$ & $28.16^{\mathrm{gh}}$ & $102.67^{\mathrm{a}-\mathrm{d}}$ & $29.85^{\mathrm{b}}$ & $0.994^{\mathrm{h}}$ \\
\hline $07 \mathrm{MW} 6035$ & $83.67^{\mathrm{c}-\mathrm{g}}$ & $140.3^{\mathrm{de}}$ & $161.7^{\mathrm{fg}}$ & $26.00^{\mathrm{c}-\mathrm{e}}$ & $43.00^{\mathrm{e}-\mathrm{g}}$ & $23.06^{\mathrm{h}}$ & $85.33^{b-f}$ & $28.14^{\mathrm{b}}$ & $0.938^{\mathrm{h}}$ \\
\hline 07MW6002 & $118.67^{\mathrm{a}}$ & $179.0^{\mathrm{a}}$ & $170.3^{\mathrm{ef}}$ & $32.33^{\mathrm{b}-\mathrm{e}}$ & $62.67^{\mathrm{a}}$ & $56.07^{\mathrm{d}-\mathrm{g}}$ & $121.00^{\mathrm{a}-\mathrm{c}}$ & $33.00^{\mathrm{b}}$ & $3.433^{b-f}$ \\
\hline Asosa_1 & $97.33^{\mathrm{a}-\mathrm{e}}$ & $157.7^{\mathrm{bc}}$ & $170.3^{\mathrm{ef}}$ & $42.33^{\mathrm{a}-\mathrm{c}}$ & $61.00^{\mathrm{ab}}$ & $84.02^{\mathrm{a}-\mathrm{d}}$ & $134.33^{\mathrm{a}}$ & $27.64^{\mathrm{b}}$ & $5.120^{\mathrm{a}}$ \\
\hline Adukara & $101.0^{\mathrm{a}-\mathrm{d}}$ & $139.0^{\mathrm{de}}$ & $146.3^{\mathrm{h}}$ & $16.67^{\mathrm{e}}$ & $46.00^{\mathrm{d}-\mathrm{f}}$ & $42.61^{\mathrm{f}-\mathrm{h}}$ & $72.67^{\mathrm{d}-\mathrm{g}}$ & $25.97^{\mathrm{b}}$ & $1.953^{\mathrm{gh}}$ \\
\hline 07MW6052 & $94.23^{\mathrm{e}-\mathrm{g}}$ & $116.5^{\mathrm{cd}}$ & $156.6^{\mathrm{fg}}$ & $32.11^{\mathrm{b}-\mathrm{e}}$ & $44.67^{\mathrm{e}-\mathrm{g}}$ & $56.43^{\mathrm{c}-\mathrm{f}}$ & $66.31^{\mathrm{e}-\mathrm{g}}$ & $32.54^{\mathrm{b}}$ & $2.231^{\mathrm{d}-\mathrm{g}}$ \\
\hline GM & 85.24 & 135.4 & 183.16 & 36.90 & 51.00 & 60.18 & 78.60 & 32.46 & 3.10 \\
\hline $\mathrm{CV} \%$ & 16.3 & 6.4 & 4.1 & 25.6 & 10.7 & 24.5 & 25.7 & 16.3 & 24.0 \\
\hline
\end{tabular}

$\mathrm{NB}$ :DF=Days to flowering(days),DM=Days to maturity(days), $\mathrm{PH}=\mathrm{Plant}$ height(cm), GFP=Grain filling period(days), $\mathrm{GFR}=$ Grain filling rate(days), $\mathrm{SCH}=$ Stand count at harvest(number), $\mathrm{HW}=\mathrm{Head}$ weight(gram), $\mathrm{HSW}=$ Hundred seed weight(gram), GY= Grain yield(ton/ha), CV=Coefficient of variation(\%), GM=Grand mean.

Table 5. Mean square values of yield and yield related traits of sorghum varieties at Miesso

\begin{tabular}{|c|c|c|c|c|c|c|c|c|}
\hline Varieties & DF & $\mathrm{PH}$ & $\mathrm{SCH}$ & GFP & GFR & HW & HSW & GY \\
\hline Baji & $87.33^{\mathrm{c}-\mathrm{e}}$ & $152.3^{\mathrm{c}-\mathrm{f}}$ & $26.33^{\mathrm{d}-\mathrm{h}}$ & $84.67^{\mathrm{a}-\mathrm{c}}$ & $15.48^{\mathrm{c}}$ & $61.00^{\mathrm{a}-\mathrm{d}}$ & $27.11^{b c}$ & $1.287^{\mathrm{cd}}$ \\
\hline Birmash & $86.00^{c-e}$ & $154.3^{\mathrm{c}-\mathrm{e}}$ & $36.00^{\mathrm{a}-\mathrm{d}}$ & $83.67^{a-c}$ & $20.89^{c}$ & $40.00^{\mathrm{c}-\mathrm{f}}$ & $30.36^{\mathrm{bc}}$ & $1.721^{\mathrm{b}-\mathrm{d}}$ \\
\hline Geremew & $102.00^{\mathrm{a}-\mathrm{c}}$ & $129.0^{\mathrm{fg}}$ & $22.33^{\mathrm{e}-\mathrm{h}}$ & $99.00^{\mathrm{a}}$ & $10.47^{\mathrm{c}}$ & $52.33^{\mathrm{a}-\mathrm{d}}$ & $28.09^{b c}$ & $1.037^{\mathrm{d}}$ \\
\hline Lalo & $94.67^{\mathrm{b}-\mathrm{e}}$ & $273.7^{\mathrm{a}}$ & $32.33^{\mathrm{b}-\mathrm{e}}$ & $38.33^{\text {gh }}$ & $44.92^{\mathrm{ab}}$ & $74.00^{\mathrm{a}}$ & $26.44^{\mathrm{bc}}$ & $1.680^{\mathrm{b}-\mathrm{d}}$ \\
\hline Teshale & $82.00^{\text {de }}$ & $185.7^{\mathrm{b}}$ & $43.67^{\mathrm{ab}}$ & $80.00^{\mathrm{a}-\mathrm{d}}$ & $31.20^{\mathrm{a}-\mathrm{c}}$ & $57.67^{\mathrm{a}-\mathrm{d}}$ & $28.74^{\mathrm{bc}}$ & $2.500^{\mathrm{a}}$ \\
\hline Melkam & $86.33^{\mathrm{c}-\mathrm{e}}$ & $139.7^{\mathrm{c}-\mathrm{g}}$ & $36.00^{\mathrm{a}-\mathrm{d}}$ & $84.33^{\mathrm{a}-\mathrm{c}}$ & $20.14^{\mathrm{c}}$ & $43.33^{\mathrm{b}-\mathrm{f}}$ & $35.48^{\mathrm{ab}}$ & $1.693^{\mathrm{b}-\mathrm{d}}$ \\
\hline Gobiye & $87.67^{\mathrm{c}-\mathrm{e}}$ & $129.7^{\mathrm{fg}}$ & $40.00^{\mathrm{a}-\mathrm{c}}$ & $85.33^{\mathrm{a}-\mathrm{c}}$ & $17.80^{c}$ & $37.33^{\mathrm{d}-\mathrm{f}}$ & $36.46^{\mathrm{ab}}$ & $1.476^{\mathrm{b}-\mathrm{d}}$ \\
\hline Abshir & $79.00^{\mathrm{e}}$ & $138.3^{\mathrm{c}-\mathrm{g}}$ & $33.00^{\mathrm{b}-\mathrm{e}}$ & $70.67^{\mathrm{a}-\mathrm{f}}$ & $22.03^{c}$ & $40.00^{\mathrm{c}-\mathrm{f}}$ & $30.51^{b c}$ & $1.571^{\mathrm{b}-\mathrm{d}}$ \\
\hline Dagim & $96.33^{\mathrm{a}-\mathrm{e}}$ & $133.0^{\mathrm{e}-\mathrm{g}}$ & $30.00^{c-f}$ & $93.33^{\mathrm{ab}}$ & $13.16^{\mathrm{c}}$ & $41.00^{\mathrm{b}-\mathrm{f}}$ & $29.83^{\mathrm{bc}}$ & $1.232^{\mathrm{cd}}$ \\
\hline IS9302 & $86.33^{c-e}$ & $153.0^{\mathrm{c}-\mathrm{f}}$ & $32.33^{\mathrm{b}-\mathrm{e}}$ & $84.00^{\mathrm{a}-\mathrm{c}}$ & $16.80^{\mathrm{c}}$ & $41.67^{\mathrm{b}-\mathrm{f}}$ & $29.21^{\mathrm{bc}}$ & $1.368^{\mathrm{cd}}$ \\
\hline ESH-1 & $80.67^{\mathrm{de}}$ & $162.3^{\mathrm{c}}$ & $35.33^{\mathrm{a}-\mathrm{d}}$ & $78.67^{\mathrm{a}-\mathrm{e}}$ & $23.74^{b c}$ & $63.00^{\mathrm{a}-\mathrm{c}}$ & $31.78^{\mathrm{bc}}$ & $1.832^{\mathrm{a}-\mathrm{d}}$ \\
\hline Birhan & $79.67^{\mathrm{e}}$ & $139.0^{\mathrm{c}-\mathrm{g}}$ & $40.00^{\mathrm{a}-\mathrm{c}}$ & $77.67^{\mathrm{a}-\mathrm{e}}$ & $21.23^{\mathrm{c}}$ & $53.33^{\mathrm{a}-\mathrm{d}}$ & $43.05^{\mathrm{a}}$ & $1.650^{\mathrm{b}-\mathrm{d}}$ \\
\hline Gambela-1107 & $93.00^{\mathrm{b}-\mathrm{e}}$ & $157.7^{\mathrm{cd}}$ & $37.67^{\mathrm{a}-\mathrm{d}}$ & $90.33^{\mathrm{ab}}$ & $19.63^{c}$ & $66.33^{\mathrm{ab}}$ & $29.60^{\mathrm{bc}}$ & $1.747^{\mathrm{b}-\mathrm{d}}$ \\
\hline Emahoy & $88.67^{\text {c-e }}$ & $197.0^{\mathrm{b}}$ & $48.00^{\mathrm{a}}$ & $86.33^{\mathrm{a}-\mathrm{c}}$ & $23.22^{\mathrm{bc}}$ & $57.67^{\mathrm{a}-\mathrm{d}}$ & $33.18^{\mathrm{ab}}$ & $1.975^{\mathrm{a}-\mathrm{c}}$ \\
\hline Dekeba & $93.33^{\mathrm{b}-\mathrm{e}}$ & $118.0^{\mathrm{g}}$ & $31.67^{\mathrm{b}-\mathrm{e}}$ & $90.67^{\mathrm{ab}}$ & $15.99^{c}$ & $71.67^{\mathrm{a}}$ & $36.07^{\mathrm{ab}}$ & $1.442^{\mathrm{b}-\mathrm{d}}$ \\
\hline Chemeda & $109.33^{\mathrm{ab}}$ & $288.3^{\mathrm{a}}$ & $32.00^{\mathrm{b}-\mathrm{e}}$ & $50.00^{\mathrm{e}-\mathrm{g}}$ & $52.39^{\mathrm{a}}$ & $62.33^{\mathrm{a}-\mathrm{d}}$ & $36.77^{\mathrm{ab}}$ & $2.200^{\mathrm{ab}}$ \\
\hline Local & $97.67^{\mathrm{a}-\mathrm{d}}$ & $137.0^{\mathrm{d}-\mathrm{g}}$ & $27.67^{\mathrm{c}-\mathrm{g}}$ & $56.33^{\mathrm{c}-\mathrm{g}}$ & $33.21^{\mathrm{a}-\mathrm{c}}$ & $73.33^{\mathrm{a}}$ & $27.56^{\mathrm{bc}}$ & $1.241^{\mathrm{cd}}$ \\
\hline $07 \mathrm{MW} 6035$ & $91.33^{\mathrm{c}-\mathrm{e}}$ & $145.0^{\mathrm{c}-\mathrm{f}}$ & $24.67^{\mathrm{d}-\mathrm{h}}$ & $64.67^{b-g}$ & $25.16^{\mathrm{bc}}$ & $49.33^{\mathrm{a}-\mathrm{e}}$ & $29.61^{b c}$ & $1.212^{\mathrm{cd}}$ \\
\hline 07MW6002 & $109.67^{\mathrm{ab}}$ & $152.0^{\mathrm{c}-\mathrm{f}}$ & $18.00^{\mathrm{f}-\mathrm{h}}$ & $59.00^{\mathrm{c}-\mathrm{g}}$ & $11.61^{\mathrm{c}}$ & $26.27^{\mathrm{ef}}$ & $25.77^{\mathrm{bc}}$ & $0.170^{\mathrm{e}}$ \\
\hline Asosa_1 & $112.00^{\mathrm{a}}$ & $156.0^{\mathrm{c}-\mathrm{e}}$ & $17.00^{\mathrm{gh}}$ & $44.67^{\mathrm{fg}}$ & $10.90^{\mathrm{c}}$ & $19.60^{\mathrm{f}}$ & $21.10^{\mathrm{c}}$ & $0.100^{\mathrm{e}}$ \\
\hline Adukara & $90.67^{\text {c-e }}$ & $117.0^{\mathrm{g}}$ & $15.00^{\mathrm{h}}$ & $53.00^{\mathrm{d}-\mathrm{g}}$ & $23.28^{\mathrm{bc}}$ & $58.67^{\mathrm{a}-\mathrm{d}}$ & $26.13^{b c}$ & $1.025^{\mathrm{cd}}$ \\
\hline 07MW6052 & $96.43^{\mathrm{a}-\mathrm{d}}$ & $156.23^{\mathrm{cd}}$ & $34.67^{\mathrm{a}-\mathrm{d}}$ & $84.65^{\mathrm{a}-\mathrm{c}}$ & $30.20^{\mathrm{a}-\mathrm{c}}$ & $56.21^{\mathrm{a}-\mathrm{d}}$ & $30.42^{b c}$ & $1.542^{\mathrm{b}-\mathrm{d}}$ \\
\hline GM & 92.08 & 159.9 & 31.38 & 74.03 & 22.54 & 51.90 & 30.61 & 1.44 \\
\hline $\mathrm{CV} \%$ & 9.6 & 7.9 & 21.3 & 20.9 & 53.2 & 25.0 & 18.7 & 28.6 \\
\hline
\end{tabular}

$\mathrm{NB}: \mathrm{DF}=$ Days to flowering(days), DM=Days to maturity(days), $\mathrm{PH}=\mathrm{Plant}$ height(cm), GFP=Grain filling 
period(days), $\mathrm{GFR}=$ Grain filling rate(days), $\mathrm{SCH}=\mathrm{Stand}$ count at harvest(number), $\mathrm{HW}=\mathrm{Head}$ weight(gram), $\mathrm{HSW}=$ Hundred seed weight(gram), GY=Grain yield(ton/ha), CV=Coefficient of variation, GM=Grand mean. Among the tested genotypes, the most early flowering were ESH-1 (73.93) followed by Birhan (77.33) and Dekeba (780.2).However, Chemeda (182.7) days followed by Asosa-1 (185.9) days had the most late flowering period. Among the tested genotypes some of them had late maturity period. The top three late maturing were Chemeda (182.7) days followed by Asosa-1(185.9), Adukara (173.7) days (Table 6).

Table 6. Combined mean square values of yield and yield related traits of sorghum varieties at Mechara and Miesso

\begin{tabular}{|c|c|c|c|c|c|c|c|c|c|}
\hline Varieties & $\mathrm{DF}$ & DM & $\mathrm{PH}$ & $\mathrm{SCH}$ & GFP & GFR & HW & HSW & GY \\
\hline Baji & $85.00^{\mathrm{c}-\mathrm{e}}$ & $143.3^{\text {cd }}$ & $166.2^{\mathrm{e}-\mathrm{g}}$ & $52.33^{\mathrm{a}-\mathrm{c}}$ & $75.00^{b}$ & $47.08^{\mathrm{bc}}$ & $73.09^{c-e}$ & $28.75^{\mathrm{e}-\mathrm{h}}$ & $3.34^{\mathrm{ab}}$ \\
\hline Birmash & $87.73^{\mathrm{cd}}$ & $135.1^{\mathrm{d}}$ & $183.7^{\mathrm{e}}$ & $53.60^{\mathrm{ab}}$ & $67.47^{\mathrm{bc}}$ & $54.26^{\mathrm{bc}}$ & $82.16^{b-d}$ & $30.93^{c-g}$ & $3.52^{\mathrm{a}}$ \\
\hline Geremew & $94.67^{\mathrm{bc}}$ & $149.5^{\mathrm{b}-\mathrm{d}}$ & $151.6^{\mathrm{f}-\mathrm{j}}$ & $49.67^{\mathrm{a}-\mathrm{d}}$ & $74.00^{\mathrm{b}}$ & $41.40^{b c}$ & $86.36^{\mathrm{a}-\mathrm{c}}$ & $27.69^{\mathrm{f}-\mathrm{h}}$ & $2.81^{\mathrm{a}-\mathrm{d}}$ \\
\hline Lalo & $114.00^{\mathrm{a}}$ & $167.2^{\mathrm{a}-\mathrm{c}}$ & $353.2^{\mathrm{a}}$ & $59.53^{\mathrm{a}}$ & $53.27^{\mathrm{bc}}$ & $62.42^{\mathrm{ab}}$ & $105.25^{\mathrm{a}}$ & $26.92^{\mathrm{gh}}$ & $3.17^{\mathrm{a}-\mathrm{c}}$ \\
\hline Teshale & $82.00^{\mathrm{de}}$ & $131.5^{\mathrm{d}}$ & $176.5^{\text {ef }}$ & $42.27^{\mathrm{b}-\mathrm{e}}$ & $67.73^{b c}$ & $41.66^{\mathrm{bc}}$ & $49.65^{\mathrm{f}-\mathrm{h}}$ & $32.75^{\mathrm{c}-\mathrm{f}}$ & $2.58^{\mathrm{a}-\mathrm{e}}$ \\
\hline Melkam & $79.60^{\mathrm{de}}$ & $130.8^{\mathrm{d}}$ & $151.3^{\mathrm{f}-\mathrm{j}}$ & $35.13^{\mathrm{e}}$ & $68.87^{\mathrm{bc}}$ & $36.13^{b c}$ & $46.47^{\mathrm{f}-\mathrm{h}}$ & $34.90^{b c}$ & $2.23^{\mathrm{c}-\mathrm{f}}$ \\
\hline Gobiye & $80.13^{\mathrm{de}}$ & $131.6^{\mathrm{d}}$ & $133.3^{\mathrm{ij}}$ & $37.47^{\mathrm{de}}$ & $69.27^{\mathrm{bc}}$ & $28.34^{b c}$ & $38.23^{\mathrm{h}}$ & $35.03^{b c}$ & $1.83^{\mathrm{d}-\mathrm{f}}$ \\
\hline Abshir & $82.00^{\text {de }}$ & $129.2^{\mathrm{d}}$ & $144.1^{\mathrm{g}-\mathrm{j}}$ & $39.33^{\mathrm{c}-\mathrm{e}}$ & $65.53^{b c}$ & $24.52^{\mathrm{c}}$ & $40.76^{\mathrm{h}}$ & $31.54^{\mathrm{c}-\mathrm{g}}$ & $1.52^{\mathrm{f}}$ \\
\hline Dagim & $87.67^{\mathrm{cd}}$ & $140.0^{\mathrm{cd}}$ & $172.5^{\text {ef }}$ & $49.40^{\mathrm{a}-\mathrm{d}}$ & $71.27^{\mathrm{bc}}$ & $42.03^{b c}$ & $60.73^{\mathrm{e}-\mathrm{g}}$ & $29.53^{\mathrm{d}-\mathrm{h}}$ & $2.79^{\mathrm{a}-\mathrm{d}}$ \\
\hline IS9302 & $84.87^{\mathrm{c}-\mathrm{e}}$ & $143.1^{\mathrm{cd}}$ & $183.7^{\mathrm{e}}$ & $56.20^{\mathrm{a}}$ & $75.53^{\mathrm{b}}$ & $44.35^{\mathrm{bc}}$ & $76.63^{\mathrm{c}-\mathrm{e}}$ & $30.67^{\mathrm{c}-\mathrm{g}}$ & $3.21^{\mathrm{a}-\mathrm{c}}$ \\
\hline ESH-1 & $73.93^{\mathrm{e}}$ & $128.7^{\mathrm{d}}$ & $180.5^{\mathrm{e}}$ & $39.40^{\mathrm{c}-\mathrm{e}}$ & $70.87^{\mathrm{bc}}$ & $30.98^{\mathrm{bc}}$ & $60.60^{\mathrm{e}-\mathrm{g}}$ & $31.56^{\mathrm{c}-\mathrm{g}}$ & $2.05^{\mathrm{d}-\mathrm{f}}$ \\
\hline Birhan & $77.33^{\mathrm{de}}$ & $129.1^{\mathrm{d}}$ & $161.6^{\mathrm{e}-\mathrm{h}}$ & $37.00^{\mathrm{de}}$ & $67.73^{b c}$ & $26.10^{b c}$ & $41.47^{\text {gh }}$ & $38.32^{\mathrm{ab}}$ & $1.66^{\mathrm{ef}}$ \\
\hline Gambela-1107 & $80.07^{\text {de }}$ & $129.2^{\mathrm{d}}$ & $179.8^{\mathrm{e}}$ & $40.07^{\mathrm{c}-\mathrm{e}}$ & $67.27^{\mathrm{bc}}$ & $47.33^{\mathrm{bc}}$ & $57.24^{\mathrm{e}-\mathrm{h}}$ & $29.87^{\mathrm{c}-\mathrm{g}}$ & $2.79^{\mathrm{a}-\mathrm{d}}$ \\
\hline Emahoy & $80.20^{\mathrm{de}}$ & $159.7^{\mathrm{a}-\mathrm{d}}$ & $217.9^{d}$ & $62.07^{\mathrm{a}}$ & $97.27^{\mathrm{a}}$ & $45.93^{b c}$ & $50.41^{\mathrm{e}-\mathrm{h}}$ & $40.86^{\mathrm{a}}$ & $3.19^{\mathrm{a}-\mathrm{c}}$ \\
\hline Dekeba & $85.07^{\mathrm{c}-\mathrm{e}}$ & $130.7^{\mathrm{d}}$ & $127.3^{\mathrm{j}}$ & $39.60^{\mathrm{c}-\mathrm{e}}$ & $65.53^{b c}$ & $40.74^{b c}$ & $56.36^{\mathrm{e}-\mathrm{h}}$ & $33.60^{\mathrm{b}-\mathrm{e}}$ & $2.27^{\mathrm{c}-\mathrm{f}}$ \\
\hline Chemeda & $121.93^{\mathrm{a}}$ & $182.7^{\mathrm{a}}$ & $311.2^{\mathrm{b}}$ & $36.40^{\mathrm{de}}$ & $62.13^{b c}$ & $41.58^{\mathrm{bc}}$ & $72.55^{\mathrm{c}-\mathrm{e}}$ & $34.66^{\mathrm{b}-\mathrm{d}}$ & $2.39^{\mathrm{b}-\mathrm{f}}$ \\
\hline Local & $115.13^{\mathrm{a}}$ & $165.7^{\mathrm{a}-\mathrm{c}}$ & $274.2^{\mathrm{c}}$ & $42.87^{\mathrm{b}-\mathrm{e}}$ & $50.00^{c}$ & $42.03^{b c}$ & $87.67^{\mathrm{a}-\mathrm{c}}$ & $38.64^{\mathrm{ab}}$ & $2.09^{\mathrm{d}-\mathrm{f}}$ \\
\hline $07 \mathrm{MW} 6035$ & $95.00^{\mathrm{bc}}$ & $152.4^{\mathrm{b}-\mathrm{d}}$ & $143.2^{\mathrm{g}-\mathrm{j}}$ & $41.07^{\mathrm{b}-\mathrm{e}}$ & $61.53^{b c}$ & $40.43^{b c}$ & $99.25^{\mathrm{ab}}$ & $28.55^{\mathrm{e}-\mathrm{h}}$ & $2.29^{\mathrm{c}-\mathrm{f}}$ \\
\hline 07MW6002 & $95.00^{\mathrm{bc}}$ & $150.7^{\mathrm{b}-\mathrm{d}}$ & $158.3^{\mathrm{e}-\mathrm{i}}$ & $41.60^{\mathrm{b}-\mathrm{e}}$ & $64.20^{\mathrm{bc}}$ & $43.92^{b c}$ & $84.71^{\mathrm{b}-\mathrm{d}}$ & $26.60^{\mathrm{gh}}$ & $2.78^{\mathrm{a}-\mathrm{d}}$ \\
\hline Asosa 1 & $123.60^{\mathrm{a}}$ & $185.9^{\mathrm{a}}$ & $159.1^{\mathrm{e}-\mathrm{i}}$ & $30.87^{\mathrm{e}}$ & $64.33^{\mathrm{bc}}$ & $26.34^{b c}$ & $58.21^{\mathrm{e}-\mathrm{h}}$ & $30.69^{\mathrm{c}-\mathrm{g}}$ & $1.56^{\mathrm{f}}$ \\
\hline Adukara & $118.67^{\mathrm{a}}$ & $173.7^{\mathrm{ab}}$ & $156.8^{\mathrm{e}-\mathrm{i}}$ & $36.53^{\mathrm{de}}$ & $54.33^{\mathrm{bc}}$ & $89.92^{\mathrm{a}}$ & $64.67^{\mathrm{d}-\mathrm{f}}$ & $31.74^{\mathrm{c}-\mathrm{g}}$ & $2.35^{\mathrm{b}-\mathrm{f}}$ \\
\hline 07MW6052 & $100.0^{\mathrm{b}}$ & $153.4^{\mathrm{b}-\mathrm{d}}$ & $137.1^{\mathrm{g}-\mathrm{j}}$ & $39.20^{\mathrm{c}-\mathrm{e}}$ & $60.87^{\mathrm{bc}}$ & $47.34^{\mathrm{bc}}$ & $85.12^{\mathrm{a}-\mathrm{d}}$ & $24.53^{\mathrm{h}}$ & $2.79^{\mathrm{a}-\mathrm{d}}$ \\
\hline GM & 92.89 & 147.42 & 182.9 & 42.95 & 67.00 & 43.71 & 67.16 & 31.74 & 2.51 \\
\hline CV\% & 14.7 & 24.0 & 17.7 & 35.2 & 39.0 & 26.0 & 37.2 & 19.5 & 26.0 \\
\hline
\end{tabular}

NB:DF=Days to flowering(days), DM=Days to maturity(days), $\mathrm{PH}=\mathrm{Plant}$ height(cm), $\mathrm{GFP}=\mathrm{Grain}$ filling period(days), $\mathrm{GFR}=$ Grain filling rate(days), $\mathrm{SCH}=\mathrm{Stand}$ count at harvest(number), $\mathrm{HW}=\mathrm{Head}$ weight $(\mathrm{gram})$, $\mathrm{HSW}=$ Hundred seed weight(gram), GY= Grain yield(ton/ha), CV=Coefficient of variation, GM=Grand mean.

Differences between GCV and PCV for the agronomic traits studied were found to be less indicating that these traits were less affected by environmental fluctuations (Table 7). This is in agreement with the results of Jalata et al. (2011) and Danquah and Ofori (2012) in vulgare landraces and accessions of garden eggplant, respectively. Desmukh et al. (1986) categorized PCV and GCV values into following classes; as high $(>20 \%)$, medium $(10-20 \%)$ and low $(<10 \%)$. In this study, PCV values ranged from $15.5 \%$ in grain yield to $96.8 \%$ in grain filling rate. The GCV ranged from $13.61 \%$ in grain yield to $89.82 \%$ in head weight (Table 7 ). Based on Desmukh et al. (1986) classification, the only agronomic trait which recorded medium value for PCV and GCV was grain yield in ton/ha. However other agronomic traits evaluated recorded values of GCV and PCV well above the medium range. This suggests sufficient genetic variability to facilitate improvement through selection of these agronomic traits. The development of effective breeding programme depends on existence of genetic variability.

Estimates of Heritability: The efficiency with which genotypic variability can be exploited by selection depends upon heritability of individual traits (Bilgin et al., 2010). In addition, it gives an indication as to how a given trait or agronomic character will respond to selection (Falconer and Mackey, 1996). In the present study, high heritability value was recorded for plant height, number of days to flowering, head weight, hundred seed weight. Plant height had the highest heritability estimate $(98 \%)$ followed by number of days to flowering (95\%) (Table 7). Grain filling rate had the lowest value (59\%) followed by grain yield (77\%). 
Table 7. Genetic parameters of yield contributing characters in 22 varieties of sorghum

\begin{tabular}{lccccccccc}
\hline Traits & MSg & MSe & GV & PV & GCV $\%$ & PCV\% & H $^{2} \mathrm{~B}$ & GM & GA \\
\hline DF & 3713.7 & 185.2 & 3651.97 & 3837.17 & 62.71 & 64.2 & 0.95 & 92.89 & 182.1 \\
DM & 5014.0 & 1255 & 4595.67 & 5850.67 & 55.84 & 62.9 & 0.78 & 147.42 & 238.5 \\
GFP & 1352.5 & 682.2 & 1125.07 & 1807.37 & 40.98 & 51.9 & 0.62 & 67.00 & 85.9 \\
GFR & 2930.0 & 1653 & 2379 & 4032 & 74.44 & 96.8 & 0.59 & 42.95 & 52.20 \\
HSW & 254.51 & 38.38 & 241.717 & 280.097 & 27.59 & 29.7 & 0.86 & 31.74 & 56.43 \\
HW & 5624.4 & 625.1 & 5416.03 & 6041.13 & 89.82 & 94.8 & 0.89 & 67.16 & 124.03 \\
PH & 50347 & 1049 & 49997.3 & 51046.3 & 16.56 & 16.7 & 0.98 & 182.9 & 369.03 \\
SCH & 1080.9 & 236.4 & 1002.1 & 1238.5 & 47.88 & 53.2 & 0.81 & 43.71 & 72.86 \\
GY & 5.13 & 1.42 & 4.66 & 6.08 & 13.61 & 15.5 & 0.77 & 2.51 & 3.96 \\
\hline
\end{tabular}

$\mathrm{MSg}=$ mean square of genotype, $\mathrm{MSe}=$ mean square of error, $\mathrm{GV}=$ Genotypic variance, $\mathrm{PV}=$ Phenotypic variance, $\mathrm{GCV}=$ Genotypic Coefficient of variance, $\mathrm{PCV}=$ Phenotypic coefficient of variance, $\mathrm{H}^{2} \mathrm{~B}=$ Broad sense Heritability, GM=Grand mean, GA=Genetic Advance.

Understanding of the interaction of characters among themselves and with the environment is of great use in plant breeding. Correlation studies provide information on the nature and extent of association between any two pairs of metric characters. Hence it could be possible to bring genetic improvement in one character by selection of the other of a pair. Most of the agronomic traits evaluated in this study showed positive and significant $(\mathrm{P}<0.05)$ correlation among themselves. For instance, there was also a positive and significant correlation between plant height and head weight (0.39), grain filling rate (0.37), yield (0.30), grain filling rate and yield (0.91), hundred seed weight and yield (0.26) (Table 8). The significant positive correlation among these traits suggests that these traits could be simultaneously improved without any compensatory negative effects. However, negative and significant correlation was observed between days to flowering and stand count $(-0.35)$, grain filling period and plant height $(-0.30)$. The negative relationship between these traits suggests that they should be improved independently.

Table 8. Phenotypic correlation coefficients among yield contributing characters in sorghum.

\begin{tabular}{|c|c|c|c|c|c|c|c|c|c|}
\hline Traits & $\overline{\mathrm{DE}}$ & $\overline{D F}$ & GFP & $\mathrm{PH}$ & $\overline{\mathrm{SCH}}$ & HW & GFR & HSW & Yld \\
\hline $\mathrm{DE}$ & 1.00 & & & & & & & & \\
\hline $\mathrm{DF}$ & $0.18^{\mathrm{ns}}$ & 1.00 & & & & & & & \\
\hline GFP & $-0.32^{\mathrm{ns}}$ & $0.01^{\mathrm{ns}}$ & 1.00 & & & & & & \\
\hline $\mathrm{PH}$ & $-0.07^{\mathrm{ns}}$ & $0.23^{\mathrm{ns}}$ & $-0.30^{*}$ & 1.00 & & & & & \\
\hline $\mathrm{SCH}$ & $-0.31 *$ & $-0.35^{*}$ & $0.08^{\mathrm{ns}}$ & $0.25^{\mathrm{ns}}$ & 1.00 & & & & \\
\hline HW & $0.04^{\mathrm{ns}}$ & $0.17^{\mathrm{ns}}$ & $-0.26^{*}$ & $0.39^{*}$ & $0.10^{\mathrm{ns}}$ & 1.00 & & & \\
\hline GFR & $0.05^{\mathrm{ns}}$ & $-0.18^{\mathrm{ns}}$ & $-0.55^{*}$ & $0.37 *$ & $0.50 *$ & $0.43 *$ & 1.00 & & \\
\hline HSW & $-0.09^{\mathrm{ns}}$ & $-0.24^{\mathrm{ns}}$ & $0.12^{\mathrm{ns}}$ & $0.12^{\mathrm{ns}}$ & $0.31 *$ & $0.01^{\mathrm{ns}}$ & $0.16^{\mathrm{ns}}$ & 1.00 & \\
\hline Yld & $-0.11^{\mathrm{ns}}$ & $-0.26^{*}$ & $-0.22^{\mathrm{ns}}$ & $0.30 *$ & $0.67 *$ & $0.44 *$ & $0.91 * *$ & $0.26 *$ & 1.00 \\
\hline
\end{tabular}

$\mathrm{DE}=$ Days to emergence, $\mathrm{DF}=$ Days to flowering, $\mathrm{GFP}=$ Grain filling period, $\mathrm{PH}=\mathrm{Plant}$ height, $\mathrm{SCH}=\mathrm{Stand}$ count at harvest, $\mathrm{HW}=$ Head weight, $\mathrm{GFR}=$ Grain filling rate, $\mathrm{HSW}=$ Hundred seed weight, $\mathrm{Yld}=$ Grain yield.

\section{Conclusion}

From the results of this study, it could be concluded that there were significant genetic variability among the released of sorghum varieties studied. Baji and Birmash were the highest yielding varieties. Most of the traits evaluated were positively associated among themselves and could be improved simultaneously. The genetic variability was more predominant among yield and yield related varieties of sorghum. It can be further concluded that characters such as number of days to flowering, grain filling period, grain filling rate, plant height, and head weight, weight of 100 seed grains, combining high GCV, heritability and GA should be considered during selection for yield in sorghum variability.

\section{ACKNOWLEDGEMENT}

I would like to thank Oromia Agricultural Institute (IQQO), Mechara Agricultural Research Center for financial support.

\section{References}

Ahmad, S.Q., Khan, S., Ghaffar, M. and Ahmad, F. 2011. Genetic Diversity Analysis for Yield and Other Parameters in Maize (Zea mays L.) Genotypes. Asian Journal of Agricultural Sciences, 3, 385-388.

Allard, R.W. 1960. Principles of plant breeding. John Wiley, New York. pp477.

Amsalu Ayana and Endashaw Bekele. 2012. Geographical patterns of morphological variation in sorghum [Sorghum bicolor (L.) Moench] germplasm from Ethiopia and Eritrea: Quantitative characters. Euphytica, 115: 91-104. 
Bilgin, O., Korkut, K. Z., Baser, I., Dalioglu, O., Ozturk, I.T., Kahraman and Balkan, A. 2010. Variation and heritability for some semolina characteristics and grain yield and their relations in Durum Wheat (Triticum Durum Desf.). World Journal of Agriculture Sciences, 6(3), 301-308.

Danquah, J. A. and Ofori, K. 2012. Variation and correlation among agronomic traits in 10 accessions of garden eggplant (Solanum gilo Raddi) in Ghana. International Journal of Science and Nature, Vol. 3(2): 373-379.

Deshmukh, S.N.N., Basu, M.S. and Reddy, P.S. 1986. Genetic variability, character association and path coefficient analysis of quantitative triats in Virginia bunch varieties of groundnut. Indian Journal of Agricultural Sciences, 56,515-518.

Ejeta, G. and Knoll, J.E. 2007. Marker-Assisted Selection in Sorghum. In: Varshney, R.K. and Tuberosa, R., Eds. Genomic-Assisted Crop Improvement: Vol. 2: Genomics Applications in Crops, Springer Publications, the Netherlands, 187-205. http://dx.doi.org/10.1007/978-1-4020-6297-1_9

Food and Agriculture Organization Crop Production Statistics (FAOSTAT) (2014). World Sorghum Production and Utilization. FAO, Rome.

Haile Desmae, Jordan, R. D. and Godwin D. I. 2016. Geographic patterns of phenotypic diversity in sorghum [Sorghum bicolor (L.) Moench] landraces from North Eastern Ethiopia. African Journal of Agricultural Research, 11(33).

Hall, A.E.1993. Is Dehydration Tolerance Relevant to Genotypic Differences in Leaf Senescence and Crop Adaptation to Dry Environments. In: Close, T.J. and Bray, E.A., Eds., Plant Responses to Cellular Dehydration during Environmental Stress, 1-10.

Jalata, Z., Ayana, A. and Zeleke, H. 2011. Variability, Heritability and Genetic Advance for Some Yield and Yield Related Traits in Ethiopian Barley (Hordeum vulgare L.) Landraces and Crosses. International Journal of Plant Breeding and Genetics, 5(1), 44-52.

Kebede, H., Subudhi, P.K., Rosenow, D.T. and Nguyen, H.T. 2001. Quantitative Trait Loci Influencing Drought Tolerance in Grain Sorghum (Sorghum bicolor L. Moench). Theoretical and Applied Genetics, 103, 266276.http://dx.doi.org/10.1007/s001220100541

Mihret Yirgu, Firew Mekbib and Setegn Gebeyehu. 2015. Genetic Gain in Lowland Sorghum [Sorghum Bicolor (L.) Moench] varieties in Ethiopia. International Journal of Horticulture and Plant Breeding Sciences, 2(1):1-13.

Ramirez, P. and Kelly, J.D. 1998. Traits Related to Drought Resistance in Common Bean. Euphytica, 99, $127-$ 136.http://dx.doi.org/10.1023/A:1018353200015

Rosenow, D.T. and Clark, L.E. 1995. Drought and Lodging Resistance for Quality Sorghum Crop. Proceedings of the 50 ${ }^{\text {th }}$ Annual Corn and Sorghum Industry Research Conference, Chicago, 6-7 December 1995, 82-97.

Rosenow, D.T., Ejeta, G., Clark, L.E., Gilbert, M.L., Henzell, R.G., Borrell, A.K. and Muchow, R.C. 1996. Breeding for Pre- and Post-flowering Drought Stress Resistance in Sorghum. In: Proceedings of the International Conference on Genetic Improvement of Sorghum and Pearl Millet, Lubbock, 22-27, September, 400-411.

Rosenow, D.T.1977. Breeding for Lodging Resistance in Sorghum. In: Loden, H.D. and Wilkinson, D., Eds. Proceedings of the $32^{\text {nd }}$ Annual Corn and Sorghum Industry Research Conference, American Seed Trade Association, Washington DC, 171-185.

Tuinstra, M.R., Grote, E.M., Goldsbrough, P.B. and Ejeta, G. 1997. Genetic Analysis of Post-Flowering Drought Tolerance and Components of Grain Development in Sorghum bicolor (L.) Moench. Molecular Breeding, 3, 439-448. http://dx.doi.org/10.1023/A:1009673126345

Xu, W.W., Subudhi, P.K., Crasta, O.R., Rosenow, D.T., Mullet, J.E. and Nguyen, H.T. 2000 Molecular Mapping of QTLs Conferring Stay green in Grain Sorghum (Sorghum bicolor L. Moench). Genome, 43, 461-469. http://dx.doi.org/10.1139/gen-43-3-461 